\title{
A geometric criterion for the finite generation of the Cox rings of projective surfaces
}

\author{
Brenda Leticia De La Rosa Navarro, J. Bosco Frías Medina, \\ Mustapha Lahyane, Israel Moreno Mejía and Osvaldo Osuna Castro
}

\begin{abstract}
The aim of this paper is to give a geometric characterization of the finite generation of the Cox rings of anticanonical rational surfaces. This characterization is encoded in the finite generation of the effective monoid. Furthermore, we prove that in the case of a smooth projective rational surface having a negative multiple of its canonical divisor with only two linearly independent global sections (e.g., an elliptic rational surface), the finite generation is equivalent to the fact that there are only a finite number of smooth projective rational curves of self-intersection -1 . The ground field is assumed to be algebraically closed of arbitrary characteristic.
\end{abstract}

\section{Introduction}

In [15], Galindo and Monserrat characterize the smooth projective surfaces $Z$ defined over an algebraically closed field $k$ with finitely generated Cox rings (see the next paragraph for the definition) by means of the finiteness of the set of integral curves on $Z$ of negative self-intersection, and the existence of a finitely generated $k$-algebra containing two $k$-algebras associated naturally to $Z$, see Theorem 1 , page 94 , in [15]. The aim of this work is to give an equivalent characterization of the finite generation of the Cox ring totally based on the geometry of the surface, and to apply the criterion to some classes of smooth projective rational surfaces, e.g., the anticanonical ones (i.e., those rational surfaces holding an effective anticanonical divisor) and the surfaces constructed in [7], [8], [13], and [14]; establishing thus the geometric nature of our characterization. For some purely algebraic features of the Cox ring of a variety, see [5], and [12].

Following $\mathrm{Hu}$ and Keel [20], the Cox (or the total coordinate) ring of a smooth projective variety $V$ defined over an algebraically closed field $k$ is the $k$-algebra

Mathematics Subject Classification (2010): Primary 14C20, 14C22, 14C40; Secondary 14C17, $14 \mathrm{E} 30$.

Keywords: Cox rings, rational surfaces, effective monoid, nef monoid, extremal surfaces. 
defined as follows:

$$
\operatorname{Cox}(V)=\bigoplus_{\left(n_{1}, \ldots, n_{r}\right) \in \mathbb{Z}^{r}} H^{0}\left(V, \mathcal{O}\left(L_{1}^{n_{1}} \otimes \cdots \otimes L_{r}^{n_{r}}\right)\right) .
$$

Here $\left(L_{1}, \ldots, L_{r}\right)$ is a basis of the $\mathbb{Z}$-module $\operatorname{Pic}(V)$ of classes of invertible sheaves on $V$ modulo isomorphisms under the tensor product, and we have assumed that the linear and numerical equivalences on the group of Cartier divisors on $V$ are the same, such assumption is satisfied for example for smooth projective rational surfaces $V$.

From the birational geometry classification of projective varieties, it is desirable to run the minimal model program (in short, Mori's program) for at least a canonical divisor. To this end, one needs to know the finite generation of the Cox rings of projective varieties; indeed, given a projective variety $X$, under some reasonable hypotheses, the Mori's program can be carried out for any divisor on $X$ if and only if the Cox ring of $X$ is finitely generated (see Prop. 3.2, p. 342, in [20]).

Henceforth, an interesting (but still) open problem is to classify theoretically and/or effectively and constructively all smooth projective rational surfaces $S$ for which the $k$-algebra $\operatorname{Cox}(S)$ is finitely generated. Masayoshi Nagata (see Theorem $4 \mathrm{a}$, page 283 , in [28]) showed that the surface $Z$ obtained by blowing up of the projective plane $\mathbb{P}^{2}$ at nine or more points in general position has an infinite number of (-1)-curves (see also [24], [21], [22], [25], [29], [27], [26] and [23] for cases when the points need not be in general position), consequently its Cox $\operatorname{ring} \operatorname{Cox}(Z)$ is not finitely generated. Here a $(-1)$-curve on $Z$ means a smooth projective curve on $Z$ of self-intersection equal to -1 . Note that in this example, the effective monoid $M(Z)$ of $Z$ is also not finitely generated, where $M(Z)$ stands for the set of elements of the Picard group $\operatorname{Pic}(Z)$ of $Z$ having at least a nonzero global section.

In this paper we mainly look for those smooth projective rational surfaces $S$ for which the finite generation of $\operatorname{Cox}(S)$ is equivalent to the finite generation of $M(S)$. Our two main results, Theorems 1 and 8 below which are derived from Theorem 21, give a partial answer. By the way, we have been informed by a referee that in the characteristic zero case, Theorem 21 was obtained in [1] using a different approach.

Theorem 1. Let $S$ be a smooth projective rational surface defined over an algebraically closed field $k$ of arbitrary characteristic such that the invertible sheaf associated to the divisor $-K_{S}$ has a nonzero global section. The following assertions are equivalent:

1) $\operatorname{Cox}(S)$ is finitely generated.

2) $M(S)$ is finitely generated.

3) S has only a finite number of (-1)-curves and only a finite number of (-2)curves.

Here $K_{S}$ denotes a canonical divisor on $S$.

Proof. It follows from Lemma 12 and Theorem 21 below.

It is well known that in the case of a rational surface, there are some criteria for the finite generation of its effective monoid that depend on the behavior of the 
self-intersection of a canonical divisor on the surface, and in some cases one need also the finiteness of the set of all $(-1)$ and $(-2)$-curves. The following theorem presents two of these criteria; its proof can be found in Prop. 4.3, p. 109, in [25]:

Theorem 2. Let $X$ be a smooth projective rational surface.

(a) If $K_{X}^{2}>0$, then $M(X)$ is finitely generated.

(b) If $K_{X}^{2}=0$, then $M(X)$ is finitely generated if and only if $X$ has only finitely many (-1)-curves.

Here, $K_{X}$ denotes a canonical divisor on $X$.

As consequences, the following two results hold:

Corollary 3. The Cox ring of a smooth projective rational surface having a canonical divisor of self-intersection larger than or equal to zero is finitely generated if and only if the set of $(-1)$-curves is finite.

Proof. Let $S$ be a smooth projective rational surface having a canonical divisor $K_{S}$ such that $K_{S}^{2}$ is nonnegative. It follows from Lemma 2.2, page 103, in [25] that the invertible sheaf associated to $-K_{S}$ has at least one nonzero global section. Henceforth, by Theorems 1 and 2 the result holds.

Corollary 4. The Cox ring of a smooth projective rational surface having a canonical divisor of self-intersection larger than zero is finitely generated.

Proof. Let $S$ be a smooth projective rational surface having a canonical divisor $K_{S}$ such that $K_{S}^{2}$ is positive. Then, there exists at least one nonzero global section of the invertible sheaf associated to $-K_{S}$ (see Lemma 2.2, page 103, in [25]). Applying Theorem 1, and item (a) of Theorem 2 we are done.

In particular, since a Del Pezzo surface is nothing but a blow-up of the projective plane at $r$ points with $r \leq 8$, we recover the following well-known result, see Theorem 3.2 , page 6 , in [3]:

Corollary 5. The Cox ring of a Del Pezzo surface is finitely generated.

Here, we remind the reader of the fact that the Picard number of a smooth projective rational surface is the rank of its Picard group. In the case of smooth projective rational surface having Picard number greater than nine, we have the following characterization of the finite generation of its Cox ring using the set of $(-2)$-curves.

Corollary 6. The Cox ring of a smooth projective rational surface whose Picard number is at least 10, and having an integral curve linearly equivalent to an anticanonical divisor, is finitely generated if and only if the set of (-2)-curves is finite and spans a linear subspace in the Picard group of codimension one.

Proof. The result follows from Theorem 3.1, page 142, in [16] and Theorem 1.

Remark 7. In Corollary 6, the condition on the Picard number is essential; otherwise, the set of $(-2)$-curves may not span a linear subspace in the Picard group 
of codimension one. For example, the blow-up of the projective plane at four points in general position does not hold any $(-2)$-curves. However, the Cox ring of a smooth projective rational surface having Picard number less than ten is always finitely generated (see Corollary 4).

Here is our second result:

Theorem 8. Let $Z$ be a smooth projective rational surface defined over an algebraically closed field $k$ of arbitrary characteristic such that the invertible sheaf associated to the divisor $-r K_{Z}$ has at least two linearly independent global sections for some positive integer $r$. The following assertions are equivalent:

1) $\operatorname{Cox}(Z)$ is finitely generated.

2) The set of smooth projective rational curves of self-intersection -1 on $Z$ is finite.

Here $K_{Z}$ denotes a canonical divisor on $Z$.

Proof. Apply Theorem 21 below and the fact that the set of $(-2)$-curves on $Z$ is finite.

\section{Preliminaries}

\subsection{General notions}

Let $S$ be a smooth projective surface defined over an algebraically closed field of arbitrary characteristic. From now on, we assume that the linear and numerical equivalences on the group of Cartier divisors on $S$ are the same. A canonical divisor on $S$, and the Picard group of $S$ will be denoted by $K_{S}$ and $\operatorname{Pic}(S)$ respectively. There is an intersection form on $\operatorname{Pic}(S)$ induced by the intersection of divisors on $S$; it will be denoted by a dot, that is, for $x$ and $y$ in $\operatorname{Pic}(S), x . y$ is the intersection number of $x$ and $y$ (see [19] and [2]).

The following result, known as the Riemann-Roch theorem for smooth projective surfaces, is stated using Serre duality.

Lemma 9. Let $D$ be a divisor on a smooth projective surface $S$ having an algebraically closed field of arbitrary characteristic as a ground field. Then the following equality holds:

$h^{0}\left(S, \mathcal{O}_{S}(D)\right)-h^{1}\left(S, \mathcal{O}_{S}(D)\right)+h^{0}\left(S, \mathcal{O}_{S}\left(K_{S}-D\right)\right)=1+p_{a}(S)+\frac{1}{2}\left(D^{2}-D \cdot K_{S}\right)$, where $\mathcal{O}_{S}(D)$ (respectively, $p_{a}(S)$ ) is an invertible sheaf associated canonically to the divisor $D$ (respectively, the arithmetic genus of $S$, that is $\chi\left(\mathcal{O}_{S}\right)-1$, where $\chi$ is the Euler characteristic function).

Here we recall some standard results, see [17], [19] and [2]. The Néron-Severi group $\operatorname{NS}(S)$ of $S$ is the quotient group of the group of Cartier divisors on $S$ modulo numerical equivalence, and it is well known that it is a free finitely generated $\mathbb{Z}$-module. An element $x$ of $\mathrm{NS}(S)$ is effective, respectively numerically effective (nef in short), if an element of $x$ is an effective, respectively numerically effective, 
divisor on $S$. Here a divisor $D$ on $S$ is nef if $D . C \geq 0$ for every integral curve $C$ on $S$. The set of effective elements of $\operatorname{NS}(S)$ is denoted by $M(S)$, it is obviously that it has an algebraic structure of a monoid, and is called the effective monoid of $S$. Now, we start with some properties which follow from a successive iterations of blowing up closed points of a smooth projective rational surface.

Lemma 10. Let $\pi^{*}: \mathrm{NS}(X) \rightarrow \mathrm{NS}(Y)$ be the natural group homomorphism on Néron-Severi groups induced by a given birational morphism $\pi: Y \rightarrow X$ of smooth projective rational surfaces. Then $\pi^{*}$ is an injective intersection-form preserving map of free abelian groups of finite rank. Furthermore, it preserves the dimensions of cohomology groups, the effective divisor classes and the numerically effective divisor classes.

Proof. See Lemma II.1 in [18].

Lemma 11. Let $x$ be an element of the Néron-Severi group $\mathrm{NS}(X)$ of a smooth projective rational surface $X$. The effectiveness or the nefness of $x$ implies the noneffectiveness of $\mathcal{K}_{X}-x$, where $\mathcal{K}_{X}$ denotes the element of $\operatorname{Pic}(X)$ which contains a canonical divisor on $X$. Moreover, the nefness of $x$ implies also that the self-intersection of $x$ is greater than or equal to zero.

Proof. See Lemma II.2 in [18].

The following result is also needed. We recall that a $(-1)$-curve, respectively a $(-2)$-curve, is a smooth rational curve of self-intersection -1 , respectively -2 .

Lemma 12. The monoid of effective divisor classes modulo linear equivalence on a smooth projective rational surface $X$ having an effective anticanonical divisor is finitely generated if and only if $X$ has only a finite number of (-1)-curves and only a finite number of (-2)-curves.

Proof. See Corollary 4.2 in [25].

\subsection{Extremal surfaces}

The set of nef elements $\operatorname{Nef}(S)$ in the Néron-Severi group $\operatorname{NS}(S)$ of a smooth projective surface $S$, it has obviously an algebraic structure of a monoid, and is called the nef monoid of $S$. We define two more submonoids, $\operatorname{Char}(S)$ and $[\operatorname{Char}(S): \operatorname{Nef}(S)]$ of $\operatorname{NS}(S)$ (see [6] and [15]), as follows:

Definition 13. With notation as above.

1) The characteristic monoid $\operatorname{Char}(S)$ of $S$ is the set of elements $x$ in $\operatorname{NS}(S)$ such that there exists an effective divisor on $\mathrm{S}$ whose associated complete linear system is base point free and whose class in $\mathrm{NS}(S)$ is equal to $x$.

2) The monoid of fractional base point free effective classes $[\operatorname{Char}(S): \operatorname{Nef}(S)]$ of $S$ is the set of elements $y$ in $\operatorname{NS}(S)$ such that there exists a positive integer $n$ with $n y \in \operatorname{Char}(S)$. 
The main properties that we are interested in regarding monoids Char $(S)$ and $[\operatorname{Char}(S): \operatorname{Nef}(S)]$ are those in the following lemma.

Lemma 14. With notation as above, the following hold:

1) $\operatorname{Char}(S)$ and $[\operatorname{Char}(S): \operatorname{Nef}(S)]$ are submonoids of $\operatorname{Nef}(S)$.

2) $\operatorname{Char}(S)$ is a subset of $[\operatorname{Char}(S): \operatorname{Nef}(S)]$.

Proof. The statement 2) follows immediately by the definitions of $\operatorname{Char}(S)$ and $[\operatorname{Char}(S): \operatorname{Nef}(S)]$, so only remains to prove 1). First, we prove that $\operatorname{Char}(S)$ is contained in $\operatorname{Nef}(S)$. Let $z$ be an element of $\operatorname{Char}(S)$. Then there exists an effective divisor $\Gamma$ on $S$ such that $z=[\Gamma]$ and $B s(|\Gamma|)=\emptyset$. By hypothesis we may assume that $|\Gamma|$ has no fixed components, and we can write $\Gamma=\sum_{i=1}^{r} \alpha_{i} \Gamma_{i}$ where $r$ is a positive integer, $\alpha_{i}$ is a nonnegative integer and $\Gamma_{i}$ is a prime divisor for each $i \in\{1, \ldots, r\}$. We consider an integral curve $H$ on $S$, and note that we may suppose that $H$ is different to $\Gamma_{i}$ for every $i \in\{1, \ldots, r\}$. Therefore, we have that $\Gamma . H \geq 0$ and this implies that $z$ belongs to $\operatorname{Nef}(S)$. Now, we prove that $\operatorname{Char}(S)$ is a submonoid of $\operatorname{Nef}(S)$. It is obvious that $\left[0_{\operatorname{Div}(S)}\right]$ is an element of $\operatorname{Char}(S)$. Moreover, if we have elements $x$ and $y$ of Char $(S)$, then $x+y \in \operatorname{Char}(S)$ because $x+y=\left[\Gamma_{x}+\Gamma_{y}\right]$ where $x=\left[\Gamma_{x}\right], y=\left[\Gamma_{y}\right], B s\left(\left|\Gamma_{x}\right|\right)=\emptyset, B s\left(\left|\Gamma_{y}\right|\right)=\emptyset$, and we have that $\Gamma_{x}+\Gamma_{y}$ is an effective divisor and $B s\left(\left|\Gamma_{x}+\Gamma_{y}\right|\right)=\emptyset$.

Second, we prove that $[\operatorname{Char}(S): \operatorname{Nef}(S)]$ is a submonoid of $\operatorname{Nef}(S)$. Let $\mathfrak{D}$ be an element of $[\operatorname{Char}(S): \operatorname{Nef}(S)]$. Then there exists a nonnegative integer $m$ such that $m \mathfrak{D} \in \operatorname{Char}(S)$. If $E$ is an effective divisor on $S$, we have that $m D \cdot E \geq 0$, where $D$ is a representative of $\mathfrak{D}$. This implies that $D . E \geq 0$ and so $\mathfrak{D} \in \operatorname{Nef}(S)$. Now, in an obvious way, we have that $\left[0_{\operatorname{Div}(S)}\right]$ is an element of $[\operatorname{Char}(S): \operatorname{Nef}(S)]$. Furthermore, let $\mathfrak{D}_{1}$ and $\mathfrak{D}_{2}$ be elements of $[\operatorname{Char}(S): \operatorname{Nef}(S)]$, and let $m_{1}$ and $m_{2}$ be positive integers such that $m_{1} \mathfrak{D}_{1}$ and $m_{2} \mathfrak{D}_{2}$ belong to $\operatorname{Char}(S)$. It follows that $\mathfrak{D}_{1}+\mathfrak{D}_{2} \in[\operatorname{Char}(S): \operatorname{Nef}(S)]$, because $m_{1} m_{2}\left(\mathfrak{D}_{1}+\mathfrak{D}_{2}\right)$ is an element of $\operatorname{Char}(S)$.

Here we define the ingredient needed for our criterion:

Definition 15. With notation as above, $S$ is extremal if the set of effective elements of the monoid of fractional base point free effective classes is equal to the set of effective elements of the nef monoid, that is, if we have the equality $[\operatorname{Char}(S): \operatorname{Nef}(S)] \cap M(S)=\operatorname{Nef}(S) \cap M(S)$.

Now, we present some examples of extremal surfaces:

Example 16. The projective plane $\mathbb{P}^{2}$ is an extremal surface. Indeed, it is well known that if $\mathcal{E}_{0}$ is the class of a line $L$ in the Néron-Severi group of $\mathbb{P}^{2}$, then $\operatorname{Nef}\left(\mathbb{P}^{2}\right)=\mathbb{Z}_{+} \mathcal{E}_{0}$. Using the facts $\operatorname{Nef}\left(\mathbb{P}^{2}\right)=M\left(\mathbb{P}^{2}\right), B s(|L|)=\emptyset$, and Lemma 14, one may obtain $\operatorname{Char}\left(\mathbb{P}^{2}\right)=\mathbb{Z}_{+} \mathcal{E}_{0}$, and this implies the equality between $\operatorname{Nef}\left(\mathbb{P}^{2}\right)$ and $\left[\operatorname{Char}\left(\mathbb{P}^{2}\right): \operatorname{Nef}\left(\mathbb{P}^{2}\right)\right]$.

Example 17. Let $X$ be the blow-up of the projective plane $\mathbb{P}^{2}$ at $r$ ordinary collinear points $p_{1}, \ldots, p_{r}$, where $r$ is a positive integer. Let $\mathcal{E}_{0}$ be the class of the strict transform of a line $L$ on $\mathbb{P}^{2}$ in the Néron-Severi group of $X$ such that $L$ does 
not contain any of the $r$ points, and let $\mathcal{E}_{i}$ be the class of the exceptional divisor corresponding to $p_{i}$ in the Néron-Severi group of $X$ for each $i \in\{1, \ldots, r\}$. Recall that the intersection form on $N S(X)$ is given by the equalities $\mathcal{E}_{0}^{2}=1, \mathcal{E}_{0} . \mathcal{E}_{i}=0$ and $\mathcal{E}_{i} \cdot \mathcal{E}_{j}=-\delta_{i j}$ for each $i, j \in\{1, \ldots, r\}$. Here $\mathcal{E}_{0}-\mathcal{E}_{i}$ is the class of the strict transform of any line $C_{i}$ on $\mathbb{P}^{2}$ that contains $p_{i}$, and does not contain the other ones, for each $i \in\{1, \ldots, r\}$. Lemma 10 implies that the classes $\mathcal{E}_{0}$ and $\mathcal{E}_{0}-\mathcal{E}_{i}$ are nef for every $i \in\{1, \ldots, r\}$. It follows that $\operatorname{Nef}(X)$ is equal to $\mathbb{Z}_{+} \mathcal{E}_{0}+\sum_{i=1}^{r} \mathbb{Z}_{+}\left(\mathcal{E}_{0}-\mathcal{E}_{i}\right)$ (see Example 4.2.24, page 42, in [10]). Moreover, from the facts that $B s(|L|)$ is empty and $B s\left(\left|C_{i}\right|\right)=\left\{p_{i}\right\}$ for every $i \in\{1, \ldots, r\}$, we obtain that $\mathcal{E}_{0}$ and $\mathcal{E}_{0}-\mathcal{E}_{i}$ are elements of $\operatorname{Char}(X)$ for each $i \in\{1, \ldots, r\}$. Finally, using Lemma 14 we conclude that $[\operatorname{Char}(X): \operatorname{Nef}(X)]=\operatorname{Nef}(X)$, and so $X$ is extremal.

Example 18. Let $\Sigma_{n}$ be the Hirzebruch surface associated to a nonnegative integer $n$. Let $\mathcal{C}_{n}$ be the class of a section $C_{n}$ of $\Sigma_{n}$ (unique if $n$ is positive) and let $\mathcal{F}$ be the class of a fibre $f$ of $\Sigma_{n}$. Recall that $\mathcal{C}_{n}$ and $\mathcal{F}$ satisfy the equalities $\mathcal{C}_{n}^{2}=-n, \mathcal{F}^{2}=0$, and $\mathcal{C}_{n} . \mathcal{F}=1$ (see Section 2 of Chapter $\mathrm{V}$ in [19]). We know that $\operatorname{Nef}\left(\Sigma_{n}\right)=\mathbb{Z}_{+}\left(\mathcal{C}_{n}+n \mathcal{F}\right)+\mathbb{Z}_{+} \mathcal{F}$ (see item 2 of Lemma 3.1 in [9]). Since $\mathcal{C}_{n}+n \mathcal{F}$ and $\mathcal{F}$ are the classes of the effective divisors $C_{n}+n f$ and $f$ respectively, and $B s\left(\left|C_{n}+n f\right|\right)$ and $B s(|f|)$ are empty, we infer that $\mathcal{C}_{n}+n \mathcal{F}$ and $\mathcal{F}$ are elements of $\operatorname{Char}\left(\Sigma_{n}\right)$, and henceforth, it follows that $\Sigma_{n}$ is an extremal surface by Lemma 14 .

The following example provides a smooth projective rational surface that is not extremal:

Example 19. Let $X$ be the blow-up of the projective plane $\mathbb{P}^{2}$ at twenty generic points lying on a smooth integral curve $Q$ of degree four. Let $F$ be equal to $\widetilde{C}+\widetilde{Q}$, where $\widetilde{C}$ and $\widetilde{Q}$ are the strict transforms of a generic conic $C$ and $Q$ respectively. Let $\mathcal{F}$ be the class of $F$ in the Néron-Severi group of $X$. The equalities $\mathcal{F} . \widetilde{C}=12$ and $\mathcal{F} . \widetilde{Q}=4$ ensure the nefness of $\mathcal{F}$. Furthermore, $B s(|n F|)$ is not empty because the fix part of $|n F|$ is equal to $\{\widetilde{Q}\}$ for every positive integer $n$ (see page 562 in [30]). Therefore, $X$ is not an extremal surface.

Remark 20. In the case of anticanonical rational surfaces, it occurs that every nef element of the Néron-Severi group of such surfaces is an effective one; indeed, this is a consequence of Lemmas 9 and 11. Therefore, an anticanonical rational surface $S$ is extremal if and only if $[\operatorname{Char}(S): \operatorname{Nef}(S)]$ is equal to $\operatorname{Nef}(S)$.

\section{The criterion}

Now we are able to state our geometric criterion:

Theorem 21. Let $S$ be a smooth projective surface defined over an algebraically closed field $k$ of arbitrary characteristic. The following assertions are equivalent:

1) The Cox ring $\operatorname{Cox}(S)$ is finitely generated.

2) S satisfies the following two properties:

i. $S$ is extremal, and

ii. the effective monoid $M(S)$ of $S$ is finitely generated. 
3) S satisfies the following two properties:

i. $S$ is extremal, and

ii. the nef monoid $\operatorname{Nef}(S)$ of $S$ is finitely generated.

Proof. First, we prove that 1) implies 2). Since the Cox ring of $S$ is finitely generated, there exist a finite number of curves of negative self-intersection on $S$, if not, let $I$ be an infinite set of curves of negative self-intersection on $S$, the $k$-algebra $k \oplus\left(\bigoplus_{C \in I} H^{0}(S, \mathcal{C})\right)$ is not finitely generated. Furthermore, we may assume that the effective monoid of $S$ is generated by the curves of negative selfintersection, and consequently, the effective monoid of $S$ is finitely generated. On the other hand, the finiteness of the Cox ring of $S$ implies that the nef monoid of $S$ is equal to the characteristic monoid of $S$ (see Corollary 7.4, page 1233, in [4]). Therefore, $S$ is extremal (see Lemma 14).

Second, the nef monoid of $S$ is equal to the dual effective monoid of $S$. Hence, the nef monoid of $S$ is finitely generated (see Proposition 3, page 250, in [11]). This proves that 2) implies 3).

Finally, we prove that 3) implies 1). Using Lemma 2.8 of [20], the $k$-algebra $\bigoplus_{\mathcal{D} \in \operatorname{Nef}(S)} H^{0}(S, \mathcal{D})$ is finitely generated because of the nef monoid of $S$ is finitely generated, and $S$ is extremal. Moreover, this $k$-algebra contains both $k$-algebras ${ }^{1}$ $\Delta(S)$ and $S^{\mathrm{bp}}(S)$, and therefore, applying Theorem 1 of [15], the result holds.

\section{Open problems}

We conclude this paper with the following questions:

1) Is it possible to determine explicitly all the extremal smooth projective surfaces? Note that if we could be able to classify the smooth projective surfaces that are extremal, then we may have excellent candidates of surfaces whose Cox rings are finitely generated.

2) As a consequence of item 2) of Lemma 14, for a smooth projective surface $S$, $\operatorname{Char}(S)$ is a subset of $[\operatorname{Char}(S): \operatorname{Nef}(S)]$. What kind of surfaces for which the equality holds between $\operatorname{Char}(S)$ and $[\operatorname{Char}(S): \operatorname{Nef}(S)]$ ?

\section{References}

[1] Artebani, M., Hausen, J. and Laface, A.: On Cox rings of K3 surfaces. Compos. Math. 146 (2010), no 4, 964-998.

[2] Barth, W., Peters, C. And Van de Ven, A.: Compact complex surfaces. Results in Mathematics and Related Areas (3), 4, Springer-Verlag, Berlin, 1984.

\footnotetext{
${ }^{1} \Delta(S)$ is the set of homogeneous elements $f$ in the Cox ring of $S$ such that $D_{n}$ is a nef element of $\operatorname{Pic}(S), K_{S} \cdot D_{n} \geq 0$, and $\operatorname{div}_{S}(f)+D_{n}$ is a prime divisor, where $n$ is the degree of $f$ (see Definition 1, page 93, of [15]). $S^{\mathrm{bp}}(S)$ is the subalgebra of $\operatorname{Cox}(S)$ given by $\bigoplus H^{0}\left(S, \mathcal{O}_{S}\left(D_{n}\right)\right)$, where the sum is taken over $n \in \mathbb{Z}^{r}$, where $r$ is the rank of $\operatorname{Pic}(S)$ such that $\left|D_{n}\right|$ has at most finitely many base points (see Definition 1, page 93, of [15]).
} 
[3] Batyrev, V. and Popov, O.: The Cox ring of a Del Pezzo surface. In Arithmetic of higher-dimensional algebraic varieties (Palo Alto, CA, 2002), 85-103. Progr. Math. 226, Birkhäuser Boston, Boston, MA, 2004.

[4] Berchtold, F. and Hausen, J.: Cox rings and combinatorics. Trans. Amer. Math. Soc. 359 (2007), no. 3, 1205-1252.

[5] Berchtold, F. and Hausen, J.: Homogeneous coordinates for algebraic varieties. J. Algebra 266 (2003), no. 2, 636-670.

[6] Campillo, A. and GonzÁlez-Sprinberg, G.: On characteristic cones, clusters and chains of infinitely near points. In Singularities (Oberwolfach, 1996), 251-261. Progr. Math. 162, Birkhäuser, Basel, 1998.

[7] Campillo, A., Piltant, O. and Reguera, A.: Cones of curves and of line bundles on surfaces associated with curves having one point at infinity. Proc. London Math. Soc. (3) 84 (2002), no. 3, 559-580.

[8] Campillo, A., Piltant, O. and Reguera, A.: Cones of curves and of line bundles "at infinity". J. Algebra 293 (2005), no. 2, 513-542.

[9] Cerda Rodríguez, J. A., Failla, G., Lahyane, M. and Osuna Castro, O.: Fixed loci of the anticanonical complete linear systems of anticanonical rational surfaces. Balkan J. Geom. Appl. 17 (2012), no. 1, 1-8.

[10] De la Rosa Navarro, B. L.: Códigos algebraico geométricos en dimensión superior y la finitud de los anillos de Cox de surperficies racionales. Ph. D. Thesis, Posgrado conjunto en Ciencias Matemáticas UNAM-UMSNH, Morelia, Mexico, 2013.

[11] Demazure, M., Pinkham, H. C. and Teissier, B.: Séminaire sur les singularités des surfaces. Lecture Notes in Math. 777, Springer, Berlin, 1980.

[12] Elizondo, E. J., Kurano, K. and Watanabe, K.: The total coordinate ring of a normal projective variety. J. Algebra 276 (2004), no. 2, 625-637.

[13] Galindo, C. And Monserrat, F.: The cone of curves associated to a plane configuration. Comment. Math. Helv. 80 (2005), no. 1, 75-93.

[14] Galindo, C. And Monserrat, F.: On the cone of curves and of line bundles of a rational surface. Internat. J. Math. 15 (2004), no. 4, 393-407.

[15] Galindo, C. and Monserrat, F.: The total coordinate ring of a smooth projective surface. J. Algebra 284 (2005), no. 1, 91-101.

[16] Harbourne, B.: Blowings-up of $\mathbb{P}^{2}$ and their blowings-down. Duke Math. J. 52 (1985), no. 1, 129-148.

[17] Harbourne, B.: Complete linear systems on rational surfaces. Trans. Amer. Math. Soc. 289 (1985), no. 1, 231-226.

[18] Harbourne, B.: Anticanonical rational surfaces. Trans. Amer. Math. Soc. 349 (1997), no. 3, 1191-1208.

[19] Hartshorne, R.: Algebraic geometry. Graduate Texts in Mathematics 52, Springer-Verlag, New York-Heidelberg, 1977.

[20] Hu, Y. And Keel, S.: Mori dream spaces and GIT. Michigan Math. J. 48 (2000), $331-348$.

[21] Lahyane, M.: Exceptional curves on rational surfaces having $K^{2} \geq 0$. C. R. Math. Acad. Sci. Paris 338 (2004), no. 11, 873-878.

[22] Lahyane, M.: Rational surfaces having only a finite number of exceptional curves. Math Z. 247 (2004), no. 1, 213-221. 
[23] Lahyane, M.: Exceptional curves on smooth rational surfaces with $-K$ not nef and of self-intersection zero. Proc. Amer. Math. Soc. 133 (2005), no. 6, 1593-1599.

[24] Lahyane, M.: Irreducibility of the $(-1)$-classes on smooth rational surfaces. Proc. Amer. Math. Soc. 133 (2005), no. 8, 2219-2224.

[25] Lahyane, M. and Harbourne, B.: Irreducibility of (-1)-classes on anticanonical rational surfaces and finite generation of the effective monoid. Pacific J. Math. 218 (2005), no. 1, 101-114.

[26] Miranda, R. And Persson, U.: On extremal rational elliptic surfaces. Math. Z. 193 (1986), no. 4, 537-558.

[27] Mori, S.: Threefolds whose canonical bundles are not numerically effective. Ann. of Math. (2) 116 (1982), no. 1, 133-176.

[28] Nagata, M.: On rational surfaces. II. Mem. Coll. Sci. Univ. Kyoto Ser. A Math. 33 (1960), no. 2, 271-293.

[29] Rosoff, J.: Effective divisor classes and blowings-up of $\mathbb{P}^{2}$. Pacific J. Math. 89 (1980), no. 2, 419-429.

[30] ZaRiski, O.: The theorem of Riemann-Roch for high multiples of an effective divisor on an algebraic surface. Ann. of Math (2) 76 (1962), no. 3, 560-615.

Received May 29, 2014.

Brenda Leticia De La Rosa Navarro: Facultad de Ciencias, Universidad Autónoma de Baja California, Km. 103 Carretera Tijuana-Ensenada, 22860, Ensenada, Baja California, México.

E-mail: brenda.delarosa@uabc.edu.mx

J. Bosco Frías Medina: Instituto de Física y Matemáticas, Universidad Michoacana de San Nicolás de Hidalgo, Edificio C-3, Ciudad Universitaria, Avenida Francisco J. Múgica s/n, Colonia Felicitas del Río, 58040, Morelia, Michoacán, México.

E-mail: boscof@ifm.umich.mx

Mustapha Lahyane: Instituto de Física y Matemáticas, Universidad Michoacana de San Nicolás de Hidalgo, Edificio C-3, Ciudad Universitaria, Avenida Francisco J. Múgica s/n, Colonia Felicitas del Río, 58040, Morelia, Michoacán, México.

E-mail: lahyane@ifm.umich.mx

Israel Moreno Mejía: Instituto de Matemáticas, Universidad Nacional Autónoma de México, Área de la Investigación Científica, Circuito Exterior, Ciudad Universitaria, Coyoacán, 04510, México D.F., México

E-mail: israel@matem.unam.mx

Osvaldo Osuna Castro: Instituto de Física y Matemáticas, Universidad Michoacana de San Nicolás de Hidalgo, Edificio C-3, Ciudad Universitaria, Avenida Francisco J. Múgica s/n, Colonia Felicitas del Río, 58040, Morelia, Michoacán, México.

E-mail: osvaldo@ifm.umich.mx

The first four authors were supported by PAPIIT IN102008 research grant and the projects from the Coordinación de la Investigación Científica de la Universidad Michoacana de San Nicolás de Hidalgo during the period 2010-2014. The second author acknowledges the financial support of Consejo Nacional de Ciencia y Tecnología under the Grant Number 339809. The authors are grateful to the referee for his/her comments and suggestions. 